$>$ La résistance bactérienne aux glycopeptides existait dans la nature préalablement à l'utilisation humaine des glycopeptides. Toutefois, un solide faisceau d'arguments suggère que c'est l'utilisation massive des antibiotiques de cette famille, en médecine et en élevage, qui en a permis l'émergence chez l'homme et chez l'animal. Ensuite, l'utilisation d'autres antibiotiques, notamment les céphalosporines de troisième génération, a concouru à l'amplification et à la dissémination du phénomène. La dissémination de la résistance aux glycopeptides est encore limitée à des entérocoques peu pathogènes mais qui peuvent être responsables d'infections graves chez des patients qui bénéficient par ailleurs de techniques sophistiquées de soins. Toutefois, le risque majeur associé aux entérocoques résistants aux glycopeptides (ERG) est celui de la dissémination des gènes de résistance à des espèces plus pathogènes et potentiellement épidémiques, notamment $S$. aureus. Ce transfert est possible et pourrait aboutir à la survenue d'épidémies difficiles à contrôler alors que l'on assiste à l'assèchement des filières de découvertes de nouvelles molécules antibiotiques. Ce risque justifie les efforts de contrôle de la dissémination des $\varepsilon R G$ en tant qu'objectif intégré à la politique d'antibiothérapie. <

\section{Histoire naturelle de la résistance transférable aux glycopeptides chez les entérocoques}

\section{David Skurnik, Nadège Bourgeois-Nicolaos, Antoine Andremont}

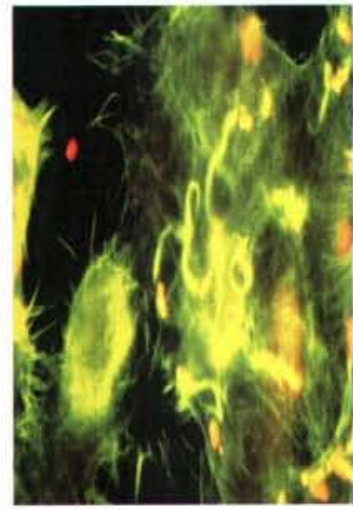

Laboratoire de Bactériologie, Hôpital

Bichat-Claude Bernard, APHP, 46, rue Henri Huchard, 75018 Paris, France. antoine.andremont@ bch,aphp.fr

Nous nous focaliserons sur les séquences d'événements, notamment les évolutions des pressions de sélection, qui ont conduit à la situation actuelle et un certain nombre d'hypothèses explicatives seront émises.

La discussion sera limitée aux deux espèces, Enterococcus faecalis et Enterococcus faecium, chez lesquelles la résistance aux glycopeptides est transférable et qui sont, à elles deux, responsables de la quasi-totalité des infections humaines à entérocoques. Deux autres espèces d'entérocoques, qui sont naturellement résistantes aux glycopeptides, $\varepsilon$. gallinarum et $\varepsilon$. casseliflavus, ne seront pas discutées. Le gène vanC qui leur confère la résistance aux glycopeptides n'est pas transférable et les infections humaines qu'elles causent sont exceptionnelles [2]. Elles sortent du cadre de cet article.

La prévalence de la résistance aux glycopeptides chez les souches d'entérocoques a augmenté de façon très importante au cours des 20 dernières années, notamment dans les hôpitaux des États-Unis. Elle est riche d'enseignements car elle est essentiellement une conséquence «écologique » de l'utilisation trop large des antibiotiques. Cet événement est un argument supplémentaire pour infléchir les stratégies d'antibiothérapie vers la réduction des consommations. Dans cet article, le détail de la génétique de la résistance des entérocoques ne sera pas décrit, des revues récentes ayant été publiées [1].
Les entérocoques : un commensal de l'homme qui pourrait être redoutable?

Les entérocoques appartiennent à un genre bactérien très largement présent au contact direct de l'homme. Le côlon est peuplé de concentrations d'entérocoques de l'ordre de 5 à $6 \log _{10} \mathrm{CFU} / \mathrm{g}$ de contenu' ${ }^{1}$ [3].

Toutefois, contrairement à Escherichia coli, lui aussi commensal du tube digestif humain, et hormis circonstances exceptionnelles, les entérocoques ne sont

${ }^{1} \mathrm{CFU}$ : colony-forming units. 
pas responsables de nombreuses infections. Apparemment, ils n'ont pas acquis, comme $\varepsilon$. coli, des gènes de pathogénicité les rendant responsables d'infections urinaires [4] ou intestinales [5]. Ils ne sont pas non plus capables de traverser la muqueuse intestinale aussi facilement qu'E. coli (ou d'autres entérobactéries) chez les patients immunodéprimés après une chimiothérapie lourde [6], pour provoquer des bactériémies. Enfin, au contraire des staphylocoques cutanés, ils ne sont pas fréquemment responsables d'infections de cathéters [7], ou de prothèses [8]. En fait, bien peu de pathologies infectieuses spécifiques sont réellement associées aux entérocoques [9]. Dans les urines, les entérocoques ne sont isolés que chez des patients ayant des facteurs de risque particuliers, comme de nombreux traitements antibiotiques antérieurs, une sonde à demeure, un diabète, une malformation ou un montage chirurgical responsable de stase [10]. Les entérocoques n'étaient pas fréquemment isolés des hémocultures chez les patients sous chimiothérapie avant les années 1980 [11]. Leur fréquence relative a augmenté après que la prise en charge antibiotique initiale ait fait diminuer chez ces patients le nombre des infections à Gram négatif qui surviennent précocement [12]. Dans les péritonites, qu'elles soient primitives ou secondaires, les entérocoques sont régulièrement isolés en raison de leur appartenance à la flore colique, mais la nécessité de diriger spécifiquement le traitement antibiotique contre eux est débattue, la pathogénie de l'infection étant plus liée, semble-t-il, aux associations bactériennes présentes dans le péritoine et aux synergies de virulence que cela amène $[13,14]$.

En fait, bien que des facteurs de virulence des entérocoques aient récemment été décrits [15] et que la fréquence des entérocoques comme cause d'endocardite soit en augmentation [16], ce n'est le plus souvent qu'en cas de diminution des défenses du patient que les entérocoques sont associés à de la pathologie. Si la question de leur résistance aux antibiotiques ne s'était pas posée, il est probable que ces véritables infections opportunistes auraient conservé un relatif anonymat en pathologie infectieuse.

\section{Une sensibilité limitée aux antibiotiques qui rend les traitements difficiles}

Jusqu'à la fin des années 1980 , c'était l'espèce $\varepsilon$. faecalis, celle qui est la plus présente au sein de l'écosystème intestinal normal, qui était logiquement la plus fréquemment isolée dans les cas d'infections humaines [2]. $\mathcal{E}$. faecalis est généralement sensible aux aminopénicillines. $\varepsilon$. faecium est moins fréquent à l'état naturel mais naturellement plus résistant, notamment aux aminopénicillines et aux uréidopénicillines. $\varepsilon$. faecium est donc avantagé par rapport à $\varepsilon$. faecalis en cas d'utilisation de ces molécules [17]. La consommation des $\beta$-lactamines a augmenté rapidement entre 1980 et 1990 [18] et il est donc logique de penser que cette augmentation a, à tout le moins, contribué au remplacement fréquent de $\varepsilon$. faecalis par $\varepsilon$. faecium $[17,19]$.

Toutefois, le phénotype sauvage de $\varepsilon$. faecium est sensible aux glycopeptides. Le simple glissement d'espèce de $\varepsilon$. faecalis à $\varepsilon$. faecium n'aboutit donc pas à une impasse thérapeutique. C'est l'apparition de la résistance aux glycopeptides, d'abord chez $\varepsilon$. faecium puis chez $\varepsilon$. faecalis, par transfert horizontal des gènes de résistance de la famille van [20], qui a amené les infections à entérocoques au premier plan de l'actualité [21]. Les possibilités thérapeutiques se sont alors réduites à deux antibiotiques récemment mis sur le marché, les synergistines injectables d'une part, mais elles ne sont actives que sur les souches de $\varepsilon$. faecium, et le linézolide d'autre part [22]. Toutefois, aucun de ces traitements n'est très facile à mettre en œuvre ni dénué de toxicité. En outre, des phénomènes de résistance à l'un ou à l'autre de ces antibiotiques ont rapidement émergé [23, 24].

Deux méta-analyses ont suggéré que la résistance aux glycopeptides était associée à une virulence accrue chez les entérocoques [25, 26]. Toutefois, les entérocoques, même résistants aux glycopeptides, restent relativement peu virulents. Le débat reste ouvert de savoir si l'isolement d'un ERG, même d'un foyer infectieux profond (comme une hémoculture) et même chez les patients les plus immunodéprimés (comme ceux atteints de leucémie ou ayant subi une greffe de moelle osseuse), ne reste pas plus un marqueur de la gravité intrinsèque de la maladie sous-jacente et des multiples traitements antibiotiques préalables qu'a reçu le patient, qu'une véritable infection ayant sa gravité propre [27].

\section{Un risque écologique majeur par rapport au risque infectieux}

Les cas d'isolements d'ERG étaient restés rares jusqu'à présent dans les hôpitaux français. Leur fréquence semble s'être accrue récemment [28]. Le risque associé n'est pas tant la mortalité car la grande majorité des patients est seulement colonisée. Le vrai risque est celui de la dissémination de la résistance aux glycopeptides chez des bactéries plus pathogènes, notamment Staphylococcus aureus résistant à la méticilline, pour lequel les glycopeptides restent le traitement de choix [29]. Le linézolide a été également proposé mais l'émergence de mutants résistants est déjà décrite [30, 31]. L'augmentation de la prévalence des ERG crée des conditions favorables à la rencontre des deux espèces, donc augmente les probabilités de transfert des gènes de résistance aux glycopeptides entre ces espèces [32]. Un tel transfert, aboutissant à des souches de $S$. aureus hautement résistantes aux glycopeptides, s'est déjà produit à plusieurs reprises aux États-Unis [20]. In vitro, la dissémination de la résistance aux glycopeptides $a$ été observée vers d'autres espèces potentiellement pathogènes comme Listeria spp [33]. Les recommandations émises aux États-Unis [34] puis en France [28] pour contrôler la dissémination des souches d'ERG visent 
bien plus à contrôler un risque écologique, celui de leur transfert de la résistance chez $S$. aureus ou d'autres pathogènes, qu'un risque médical direct, celui de la gravité des infections à entérocoques résistants.

\section{Les sources de la résistance aux glycopeptides ou l'origine des gènes}

Comme pour d'autres mécanismes de résistance, la source des gènes de résistance aux glycopeptides se trouverait chez les microorganismes producteurs de ces antibiotiques de façon naturelle [35], chez qui ils auraient évolué depuis des centaines de milliers, voire des millions d'années. Toutefois, l'homologie génétique n'est pas parfaite. L'homologie des séquences d'acides aminés varie de 54 à $61 \%$ pour les produits des gènes vanH, et de 59 à $63 \%$ pour ceux des gènes vanX, entre les souches productrices de glycopeptides dans la nature et les souches isolées chez l'homme [29]. Ces homologies ne semblent pas suffisantes pour impliquer que le transfert des micro-organismes producteurs aux souches cliniques s'est fait de façon récente au moment de l'isolement des premières souches cliniques [21], il y a une vingtaine d'années. Des intermédiaires ont donc probablement existé. Des homologues des gènes de résistance aux glycopeptides sont présents chez diverses espèces d'entérocoques de l'environnement, comme $\varepsilon$. faecium, $\varepsilon$. casseliflavus, $\varepsilon$. mundtii, $\varepsilon$. faecalis, et $\varepsilon$. durans [36], ou chez des bactéries du sol, par exemple appartenant aux genres Paenibacillus ou Rhodococcus [37], ou chez d'autres espèces de bactéries intestinales [21]. L'hypothèse peut donc être émise selon laquelle le transfert s'est fait pas à pas, d'une espèce à une autre, jusque chez les entérocoques présents chez les patients, quand la pression de sélection s'est amplifiée.

\section{Quelle pression de sélection a fait émerger les ERG ?}

Le rôle de l'exposition aux glycopeptides dans l'émergence des ERG est loin d'être complètement élucidé et compris. Les glycopeptides jouent un rôle important dans l'émergence des ERG après administration orale [38] mais leur rôle après administration parentérale (beaucoup plus fréquente) est moins évident. L'élimination intestinale des glycopeptides est faible [39] mais augmente avec la durée de la thérapeutique [40]. Après 5 jours de traitement (par $1 \mathrm{~g}$ toutes les 12 heures) elle s'échelonne de 2 à $95 \mu \mathrm{g} / \mathrm{ml}$ de selles, ce qui pourrait être suffisant pour sélectionner des $E R G$ puisque les CMI des souches d'entérocoques sensibles aux glycopeptides sont inférieures à $4 \mathrm{mg} / \mathrm{l}$ et celles des souches résistantes porteuses des gènes vanA ou van $B$ peuvent croître jusqu'à $1000 \mathrm{mg} / \mathrm{l}$. Toutefois, cela n'explique pas pourquoi, dans les années 1990, l'épidémie d'ERG a explosé dans les services de réanimation aux États-Unis mais pas en Europe [41]. On aurait pu s'attendre à un schéma inverse car, en Europe, l'avoparcine, un glycopeptide conférant une résistance croisée avec ceux utilisés chez l’homme, était largement utilisée comme promoteur de croissance, donc par voie orale, dans les élevages, alors qu'elle ne l'était pas aux États-Unis [42]. Les souches d'ERG étaient d'ailleurs prévalentes dans la population européenne par transfert à partir des animaux [43], notamment par l'intermédiaire des viandes contaminées [44], alors qu'elles ne l'étaient pas dans la population communautaire américaine [45]. Tout ceci implique qu'aux ÉtatsUnis, c'est essentiellement l'utilisation hospitalière des antibiotiques qui a été la cause de l'émergence des $\varepsilon R G$. Toutefois, pour comprendre complètement le phénomène, il faut en outre analyser comment et pourquoi ces glycopeptides ont été utilisés.

La comparaison de la consommation des glycopeptides dans les années 1980 entre hôpitaux européens (où elle était relativement faible) et américains (où elle était déjà très élevée) suggère une explication aux différences épidémiologiques observées entre l'Europe et les États-Unis : la surconsommation américaine par rapport à l'Europe a touché non seulement les glycopeptides parentéraux, mais aussi, et même surtout, les glycopeptides oraux, dont l'utilisation en Europe est restée anecdotique en médecine humaine [46]. L'explication de cette différence reste hypothétique. Les indications des glycopeptides oraux sont limitées. Dans les années 1980, c'était le traitement de choix des colites post-antibiotiques à Clostridium difficile [47]. II est donc probable que la surconsommation américaine de glycopeptides oraux chez l'homme, par rapport à l'Europe, était en rapport avec cette indication.

Aucun élément objectif n'indique que la pathologie à C. difficile était alors plus répandue aux États-Unis [48] qu'en Europe. Toutefois, le risque pour les médecins d'être attaqués pour mal-practice lorsqu'un événement indésirable survient chez leurs patients [49, 50] y est plus élevé. II est donc possible: (1) que les glycopeptides par voie orale aient été prescrits pour des cas mineurs ou simplement suspectés d'infections à $C$. difficile, pour prévenir l'évolution vers des formes graves de diarrhées post-antibiotiques qui pourraient faire l'objet de litiges; et (2) que cette sur-prescription soit la cause première de l'émergence des ERG dans les hôpitaux des États-Unis. Les prescriptions inappropriées de vancomycine par peur de la mal-practice y ont été rapportées, en néphrologie [51].

II reste à expliquer pourquoi les ERG sélectionnés en Europe chez les animaux en raison de l'utilisation d'avoparcine, et qui disséminaient chez les sujets sains, ne causaient pas de nombreuses infections humaines. II est possible que les souches d'ERG responsables d'infections humaines soient différentes des souches animales sélectionnées par l'avoparcine, ce qui aurait constitué un obstacle à la survenue d'infections, même chez les sujets colonisés par des souches animales [52].

La pression de sélection due aux glycopeptides n'est pas la seule à être en cause dans l'émergence des ERG. $\varepsilon$ n effet, les $\varepsilon$. faecium sont résistants à de nombreux 
antibiotiques, et en particulier aux $\beta$-lactamines à spectre large (céphalosporines de troisième génération, amino- et uréïdo-pénicillines avec ou sans inhibiteurs de $\beta$-lactamases). La consommation de ces antibiotiques sélectionne donc les ERG. Les céphalosporines de troisième génération sont retrouvées comme facteur de risque de colonisation et d'infection par les ERG dans de nombreuses études $[53,54]$ avec des risques relatifs qui sont même plus élevés que ceux des glycopeptides. Toutefois, l'événement premier ayant sélectionné la résistance aux glycopeptides ne peut être que l'utilisation des glycopeptides, car bien évidemment, s'il n'y a pas d'ERG, les céphalosporines de troisième génération ne seront pas un facteur de risque. Les phénomènes de co-résistance ne peuvent avoir joué qu'un rôle d'amplification favorisant: (1) l'augmentation des concentrations fécales chez un individu déjà colonisé ; et (2) la dissémination des souches et la colonisation d'autres patients. Ce rôle est particulièrement important lorsque la situation épidémique permet facilement la sélection des ERG, c'est-à-dire lorsque la pression de colonisation est importante quand de nombreux patients sont déjà porteurs d'ERG dans l'unité d'hospitalisation.

\section{Les $\varepsilon R G$ vont-ils disparaître si la pression de sélection par les glycopeptides diminue?}

Le portage d'ERG dans la population européenne semble diminuer depuis l'arrêt de l'utilisation de l'avoparcine en élevage [43]. Aux États-Unis, la prévalence des ERG au sein des entérocoques se stabilise, tandis que la consommation des glycopeptides diminue $[46,55]$. Cependant, le retrait de l'avoparcine dans les élevages européens n'y a pas fait disparaître les ERG [56], même si ceux-ci sont moins souvent isolés dans les élevages qui n'ont jamais reçu d'avoparcine [57].

Cette persistance de la résistance serait expliquée, au moins en partie, par un système plasmidique toxine-antitoxine jouxtant vanA et indispensable à la survie bactérienne chez les souches aviaires d'ERG. Ce système maintiendrait la résistance chez les souches qui le possèdent et expliquerait leur persistance chez les souches aviaires [58]. Très récemment, il a été montré que des systèmes identiques étaient également très répandus chez les souches humaines, ce qui pourrait également contribuer à la persistance de la résistance à la vancomycine chez l'homme $[58,59]$, une fois qu'elle est apparue.

Outre ces systèmes, la pression de sélection par les céphalosporines de troisième génération contribue probablement aussi à maintenir les ERG. Les données de surveillance montrent que l'augmentation de leur consommation a été forte en France ces dernières années (www.esac. ua.ac.be). C'est elle qui pourrait être à l'origine de l'augmentation du nombre de cas d'isolement des ERG en France actuellement [28]. $\diamond$

\section{SUMMARY}

\section{Natural history of glycopeptides resistance in enterococci}

Glycopeptide-resistance has not been created by the human use of antibiotics. The van gene family, which confers high levels of resistance in human enterococci is also found in micro-organisms which produce glycopeptide naturally, and in several environmental and commensal bacterial species. Glycopeptide-resistant enterococci (GRE) have emerged in humans because of the use of glycopeptide antibiotics, particularly after oral use. Heavy use of other antibiotics, such as extended spectrum beta-lactams, has massively contributed to the dissemination of GRE. GRE are not highly pathogenic but their diffusion is associated with the risk of transfer of glycopeptide resistance to pathogenic species resistant to most other antibiotics such as methicillin-resistant Staphylococcus aureus. This ecological risk is the most important rationale for controlling emergence and diffusion of GRE. $\nabla$

\section{RÉFÉRENCES}

1. Reynolds PE, Courvalin P. Vancomycin resistance in enterococci due to synthesis of precursors terminating in D-alanyl-D-serine. Antimicrob Agents Chemother $2005 ; 49: 21-5$.

2. George RC, Uttley AH. Susceptibility of enterococci and epidemiology of enterococcal infection in the 1980s. Epidemiol Infect $1989 ; 103: 403-13$

3. Drasar B, Hill M. The distribution of bacterial flora in the intestine. In : Press A, ed. Human intestinal flora, chapitre 3. London: Academic Press, $1974: 6-43$.

4. Johnson JR, Russo TA. Molecular epidemiology of extraintestinal pathogenic (uropathogenic) Escherichia coli. Int J Med Microbiol 2005 ; $295: 383-404$

5. Garmendia J, Frankel G and Crepin VF. Enteropathogenic and enterohemorrhagic Escherichia coli infections: translocation, translocation, translocation. Infect Immun 2005 ; 73 : 2573-85.

6. Tancrede $\mathrm{CH}$, Andremont AO. Bacterial translocation and Gram-negative bacteremia in patients with hematological malignancies. J Infect Dis $1985 ; 152: 99-103$.

7. Donelli G, Guaglianone $\varepsilon$. Emerging role of Enterococcus spp in catheterrelated infections: biofilm formation and novel mechanisms of antibiotic resistance. J Vasc Access 2004 ; 5 : 3-9.

8. Raymond NJ, Henry J, Workowski KA. Enterococcal arthritis: case report and review. Clin Infect Dis 1995; $21: 516-22$.

9. Pillar CM, Gilmore MS. Enterococcal virulence-pathogenicity island of E. faecalis. Front Biosci $2004 ; 9: 2335-46$.

10. Ronald A. The etiology of urinary tract infection: traditional and emerging pathogens. Am J Med 2002; 113 (suppl 1A) : \$14-9.

11. Cherif H, Kronvall G, Bjorkholm M, Kalin M. Bacteraemia in hospitalised patients with malignant blood disorders: a retrospective study of causative agents and their resistance profiles during a 14-year period without antibacterial prophylaxis. Hematol/ $2003 ; 4: 420-6$.

12. Oppenheim $B A$. The changing pattern of infection in neutropenic patients. J Antimicrob Chemother 1998; 41 (suppl D) : 7-11.

13. Montravers $P$, Andremont $A$, Massias L, Carbon C. Investigation of the potential role of Enterococcus faecalis in the pathophysiology of experimental peritonitis. J Infect Dis $1994 ; 169: 821-30$.

14. Rotstein OD, Kao J, Houston K. Reciprocal synergy between Escherichia coli and Bacteroides fragilis in an intra-abdominal infection model. I Med Microbiol 1989; 29:269-76.

15. Tsigrelis C, Singh KV, Coutinho TD, et al. Vancomycin-resistant Enterococcus faecalis endocarditis: linezolid failure and strain characterization of virulence factors. J Clin Microbiol $2007 ; 45$; $631-5$.

16. Hoen B. Special issues in the management of infective endocarditis caused by Gram-positive cocci. Infect Dis Clin North Am 2002; $16: 437-52$.

17. Huycke MM, Sahm DF, Gilmore MS. Multiple-drug resistant enterococci: the nature of the problem and an agenda for the future. Emerg Infect Dis $1998 ; 4: 239-49$.

18. Guillemot D, Maison P, Carbon C, et al. Trends in antimicrobial drug use in the community-France, 1981-1992.J Infect Dis 1998; $177: 492-7$.

19. Boyce JM, Opal SM, Potter-Bynoe G, et al. Emergence and nosocomial transmission of ampicillin-resistant enterococci. Antimicrob Agents Chemother $1992 ; 36: 1032-9$. 
20. Courvalin P. Vancomycin resistance in gram-positive cocci. Clin Infect Dis 2006; 42 (suppl 1): S25-34.

21. Leclercq R, Derlot $\varepsilon$, Duval J, Courvalin P. Plasmid-mediated resistance to vancomycin and teicoplanin in Enterococcus faecium. N Engl J Med 1988; 319:157-61.

22. Zirakzadeh A, Patel R. Vancomycin-resistant enterococci: colonization, infection, detection, and treatment. Mayo Clin Proc $2006 ; 81$ : 529-36.

23. Donabedian SM, Perri MB, Vager D, et al. Quinupristin-dalfopristin resistance in Enterococcus faecium isolates from humans, farm animals, and grocery store meat in the United States. J Clin Microbiol $2006 ; 44: 3361-5$.

24. Seedat J, Zick G, Klare I, et al. Rapid emergence of resistance to linezolid during linezolid therapy of an Enterococcus faecium infection. Antimicrob Agents Chemother 2006 ; $50: 4217-9$.

25. DiazGranados CA, Zimmer SM, Klein M, Jernigan JA. Comparison of mortality associated with vancomycin-resistant and vancomycin-susceptible enterococcal bloodstream infections: a meta-analysis. Clin Infect Dis $2005 ; 41$ : 327 - 33.

26. Salgado CD, Farr BM. Outcomes associated with vancomycin-resistant enterococci: a metaanalysis. Infect Control Hosp Epidemiol $2003 ; 24: 690-8$.

27. Dubberke ER, Hollands JM, Georgantopoulos P, et al. Vancomycin-resistant enterococcal bloodstream infections on a hematopoietic stem cell transplant unit: are the sick getting sicker? Bone Marrow Transplant 2006; 38: 813-9.

28. Leclercq $R$, Coignard $B$. Les entérocoques résistants aux glycopeptides : situation en france en 2005. BEH 2006; $13: 85-7$.

29. French $G$. Review of new guidelines for prophylaxis and treatment of MRSA infections. BrJ Hosp Med (Lond) 2006; 67:482-6.

30. Tsakris A, Pillai SK, Gold HS, et al. Persistence of rRNA operon mutated copies and rapid reemergence of linezolid resistance in Staphylococcus aureus.) Antimicrob Chemother 2007 ; $60: 649-51$.

31. Meka VG, Pillai SK, Sakoulas G, et al. Linezolid resistance in sequential Staphylococcus aureus isolates associated with a T2500A mutation in the 23S rRNA gene and loss of a single copy of rRNA. J Infect Dis 2004; $190: 311-7$.

32. Warren DK, Nitin A, Hill C, et al. Occurrence of co-colonization or co-infection with vancomycin-resistant enterococci and methicillin-resistant Staphylococcus aureus in a medical intensive care unit. Infect Control Hosp Epidemiol 2004; 25: 99-104.

33. De Niederhausern S, Sabia C, Messi P, et al. Glycopeptide-resistance transferability from vancomycin-resistant enterococci of human and animal source to Listeria spp. Lett Appl Microbiol $2004 ; 39: 483-9$.

34. Siegel JD, Rhinehart $\varepsilon$, Jackson M, Chiarello L. Management of multidrug-resistant organisms in Healthcare settings, 2006. Atlanta (GA): Centers for Disease Control and Prevention, 2006: $74 \mathrm{p}$. (412 références).

35. Marshall CG, Lessard IA, Park I, Wright GD. Glycopeptide antibiotic resistance genes in glycopeptide-producing organisms. Antimicrob Agents Chemother $1998 ; 42 ; 2215-20$.

36. Guardabassi L, Dalsgaard A. Occurrence, structure, and mobility of Tn1546-like elements in environmental isolates of vancomycin-resistant enterococci. Appl Environ Microbiol 2004 ; $70: 984-90$.

37. Guardabassi L, Perichon B, van Heijenoort J, et al. Glycopeptide resistance vanA operons in Paenibacillus strains isolated from soil. Antimicrob Agents Chemother 2005; 49:4227-33.

38. Van der Auwera P, Pensart N, Korten V, et al. Influence of oral glycopeptides on the fecal flora of human volunteers: selection of highly glycopeptide-resistant enterococci.J Infect Dis $1996 ; 173: 1129-36$.

39. Carmeli y, Samore MH, Huskins C. The association between antecedent vancomycin treatment and hospital-acquired vancomycin-resistant enterococci; a meta-analysis. Arch Intern Med $1999 ; 159 ; 2461-8$.

40. Currie BP, Lemos-Filho L. Evidence for biliary excretion of vancomycin into stool during intravenous therapy: potential implications for rectal colonization with vancomycin-resistant enterococci. Antimicrob Agents Chemother $2004 ; 48 ; 4427$-9.

41. Andremont A. Un exemple de résistance liée à la pression de sélection à l'hôpital: l'entérocoque. Journée de Pharmacologie Clinique de l'Hôpital Claude Bernard. Paris: Arnette-Blackwell, $1996: 41-9$.

42. Wegener HC, Aarestrup FM, Jensen LB, et al. Use of antimicrobial growth promoters in food animals and Enterococcus faecium resistance to therapeutic antimicrobial drugs in Europe. Emerg Infect Dis $1999 ; 5: 329-35$.
43. Klare I, Badstubner D, Konstabel C, et al. Decreased incidence of VanAtype vancomycin-resistant enterococci isolated from poultry meat and from fecal samples of humans in the community after discontinuation of avoparcin usage in animal husbandry. Microb Drug Resist 1999; 5: 45-52.

44. Gambarotto K, Ploy MC, Dupron F, et al. Occurrence of vancomycinresistant enterococci in pork and poultry products from a cattle-rearing area of France. J Clin Microbiol 2001:39:2354-5.

45. McDonald LC, Kuehnert MJ, Tenover FC, Jarvis WR. Vancomycin-resistant enterococci outside the health-care setting: prevalence, sources, and public health implications. Emerg infect Dis 1997; 3: 311-7.

46. Bonten MJ, Willems R, Weinstein RA. Vancomycin-resistant enterococci: why are they here, and where do they come from? Lancet Infect Dis $2001 ; 1: 314-25$.

47. Gross MH. Management of antibiotic-associated pseudomembranous colitis. Clin Pharm 1985; 4:304-10.

48. Archibald LK, Banerjee SN, Jarvis WR. Secular trends in hospital-acquired Clostridium difficile disease in the United States, 1987-2001.J Infect Dis $2004 ; 189: 1585-9$

49. Fink S. Rectal examinations and appendicitis malpractice claims. South Med) $1995 ; 88: 1279$

50. Davey PG, Vacani P, Parker SE, Malek MM. Assessing cost effectiveness of antimicrobial treatment: monotherapy compared with combination therapy. Eur / Surg 1994; 573 (suppl) : 67-72.

51. Golper TA, Schulman G, D'Agata EM. Indications for vancomycin in dialysis patients. Semin Dial $2000 ; 13: 389-92$.

52. $Y_{u} \mathrm{HS}$, Lee JC, Kang HY, et al. Changes in gene cassettes of class 1 integrons among Escherichia coli isolates from urine specimens collected in Korea during the last two decades. J Clin Microbiol 2003; 41:5429-33.

53. Rice LB. Emergence of vancomycin-resistant enterococci. Emerg Infect Dis $2001 ; 7: 183-7$.

54. Rice LB. Antibiotics and gastrointestinal colonization by vancomycinresistant enterococci. Eur) Clin Microbiol Infect Dis $2005 ; 24: 804-14$

55. Shlaes DM, Projan SJ, Edwards JE Jt. Antibiotic discovery: state of the state. Overcoming our vulnerability because of the downturn in corporate antibacterial R\&D could depend on regulatory and legistative reforms. ASM News $2004 ; 70 \div 275-281$.

56. Sorum $M$, Johnsen PJ, Aasnes B, et al. Prevalence, persistence, and molecular characterization of glycapeptide-resistant enterococci in Norwegian poultry and poultry farmers 3 to 8 years after the ban on avoparcin. Appl Environ Microbiol 2006; 72 : 516-21.

57. Borgen K, Simonsen GS, Sundsfjord A, et al. Continuing high prevalence of VanA-type vancomycin-resistant enterococci on Norwegian poultry forms three years after avoparcin was banned. / Appl Microbiol 2000 ; $89: 478-85$.

58. Sletvold $H$, Johnsen PI, Simonsen GS, et al. Comparative DNA analysis of two vanA plasmids from Enterococcus faecium strains isolated from poultry and a poultry farmer in Norway. Antimicrob Agents Chemother $2007 ; 51: 736-9$.

59. Moritz EM, Hergenrother PJ. Toxin-antitoxin systems are ubiquitous and plasmid-encoded in vancomycin-resistant enterococci. Proc.Nati Acad Sci USA 2007; 104:311-6.
TIRÉS À PART

A. Andremont 